\title{
Neuausgaben einiger alter Kartenwerke
}

Von Franz Flury, Niedermuhren

\section{Landtafeln des Johann Stumpf}

Hier handelt es sich um die Karten, welche der aus Bruchsal stammende Autor seiner berühmten Schweizerchronik beigelegt hatte und die von seinem Verleger Christoph Froschauer 1552 separat in Form eines Atlas herausgegeben wurden. Der Autor folgte in seiner Chronik geographischen Gesichtspunkten. Das Neue war, daß die Geschichte der Eidgenossen nicht mehr, wie es auch Stumpf selbst vorher getan hatte, in die Reichsgeschichte eingebaut wurde, sondern die Schweizer als alte, selbständige, von den Franzosen und Deutschen streng zu unterscheidende Nation erschienen. Es drückte sich da das seit den Burgunder- und dem Schwabenkriege mächtig erstarkte Nationalgefühl der Eidgenossen aus. Diese Stellungnahme ist auch beim Atlas selbst durchgeführt und es zeigte sich, daß der Atlas des Johann Stumpf, welcher nicht nur der erste Atlas der Schweiz, sondern überhaupt der erste Atlas eines Staates war, dieser Stimmung in weitem Maße entsprach, indem er jahrzehntelang neu aufgelegt werden mußte, bis ihn schließlich bessere Karten verdrängten. Wie schon die Untersuchungen von Rudolf Wolf und auch die Verzerrungsgitter von Prof. Imhof zeigen, sind die Karten von der ersten Tschudikarte von 1538 abgeleitet, enthalten jedoch wesentliche Ergänzungen gegenüber Tschudi, die von dem gelehrten Verfasser stammen, der in Kartenaufnahme und Kartenzeichnung wohl erfahren war. Der Atlas wurde 1942 von Prof. Dr. Leo Weisz mit einem illustrierten Kommentar bei Kümmerly \& Frey in Bern neu herausgegeben.

Auf dem Titelblatt der Tafeln figuriert eine Weltkarte. Leider ist die Zeichnung nicht einwandfrei, jedoch zeigt sich, daß die Radien der Parallelkreise aus einem Punkt auf dem Mittelmeridian gezogen sind, der außerhalb des Polbildes liegt. Soweit es festgestellt werden kann, sind die Abstände der Meridiane auf dem Äquator gleich den Abständen der Parallelkreise auf dem Mittelmeridian, die unter sich gleich sind, und die Abstände der Meridiane auf den verschiedenen Parallelkreisen sind gleich den entsprechenden auf der Kugel. Es handelt sich also um eine unechte Kegelprojektion, die wir heute als Bonnesche Projektion bezeichnen und die aus der zweiten, homeoteren Projektion des Ptolemäus, welche Bernard de Sylva 1511 für eine venetianische Ptolemäusausgabe verwendet 
hatte, durch Apian (Peter Bienewitz), Orontius Finäus und anderen entwickelt wurde. Ein Spezialfall der Bonneschen Projektion ist die kordiforme Projektion nach Stab-Werner, bei der das Zentrum der Parallelkreise mit dem Polbild zusammenfällt. Aus dem Vorstehenden ergibt sich, daß es sich bei der vorliegenden Weltkarte nicht um die kordiforme Projektion von Stab-Werner handelt, wie es R. Grob (Geschichte der schweizerischen Kartographie) behauptet. Das zeigt schon der erste Blick. Nebenbei sei bemerkt, daß die Bonnesche Projektion die Grundlage der Dufour- und Siegfriedkarte bildet und daß sie flächentreu ist. Die besprochene Weltkarte ist mit Nord oben orientiert.

Auf den drei folgenden Karten wird die Schweiz, Helvetia, hervorgehoben und lokalisiert im Verhältnis zu ihrer Umgebung, durch rote Umrahmung auf der Karte von Europa, durch eine wehende rote Fahne mit dem weißen Kreuz auf den Karten von Deutschland und Frankreich. Außer der Weltkarte sind alle Karten des Atlas mit Süd nach oben orientiert. Die scharfe Abtrennung der Eidgenossenschaft nicht nur von Frankreich, sondern auch von Deutschland in dieser frühen Zeit ist nicht ohne Reiz zu einer Zeit, wie derjenigen kurz vor und während des letzten Krieges, wo sogar nichtnationalsozialistische deutsche Professoren uns weismachen wollten, die Schweiz gehöre zum sogenannten geographischen Begriff Deutschland (siehe die gründlichen Auseinandersetzungen von Prof. Dr. Nußbaum, Bern, mit seinen ennetrheinischen Kollegen im "Schweizer Geograph"). Es folgen nun noch eine Übersichtskarte der Schweiz und acht Karten von Teilen der Schweiz, Gaukarten. Der Maßstab der Schweizerkarte ist rund 1:900 000 (die für die Maßstäbe in der Neuausgabe angegebenen Zahlen beruhen auf den Messungen von Rudolf Wolf). Die Schweizer und die Gaukarten wurden in der vorliegenden Reproduktion mit dem Kolorit der guten Ausgaben versehen; demgemäß weist die Schweizerkarte dunkelgraue Schrift, blaue Gewässer und einen grünen Ton auf. Die Orte sind durch hübsche Ansichten bezeichnet. Die Beschriftung ist deutlich sichtbar, die Berge sind symbolisch zwischen den Flüssen als Hügelreihen dargestellt, große Wälder durch ein besonderes Grün hervorgehoben. Nun folgt die mit Thurgau bezeichnete Karte, Darstellung analog derjenigen der Schweizerkarte, nur ausführlicher. Der eigentliche Ortspunkt wird auf den Ortsansichten mit einem rot ausgefüllten Kreis bezeichnet. Hier findet eine Farbe mehr Verwendung, nämlich ein Braun für die Berge. Die andern Gaukarten sind gleich ausgeführt, die Karte des Zürichgaus erstreckt sich von Silenen bis nach 
Eglisau und von Weesen bis nach Immensee, Maßstab etwa $1: 400000$. Im Maßstab von rund 1:600 000 ist das Ergow dargestellt; das dargestellte Gebiet erstreckt sich von Grimsel-Furka bis nach Laufenburg und von Weggis bis ins Sibental (Simmental). Das Blatt Wiflispurgergow im Maßstab etwa 1550000 erstreckt sich von Älen (Aigle) bis Ponterlin (Pontarlier) und von Unterseen-Interlaken bis Genf, umfaßt also vollständig die Kantone Freiburg und Waadt, einen großen Teil des Kantons Bern und des Kantons Neuenburg und einen Teil des Kantons Genf samt dem Genfer See. Das Blatt Die Lepontier, Maßstab etwa $1: 430$ 000, reicht von Palenza (Pallanza) bis Engelberg, vom Splügen bis Crevola. Locarno heißt Luggaris, Lugano Lowertz und das Vallis Vegetia, das Centovalli, das Kämifegertal. Das Blatt umfaßt den Tessin, und wir finden darauf Grimsel, St. Gotthard, Lukmanier, Rheinwald, Adula und den Lacus Verbanus, den Langensee. Das Blatt Rhetia, Maßstab etwa 1 : 730 000, zeigt den Kanton Graubünden und erstreckt sich von Chum (Como) bis an den Bodensee, von Finstermünz bis zum Lukmanier, zeigt auch den Bregenzerwald, Zürichsee. Walensee und Comer See. Blatt Wallis, Maßstab etwa $1: 470000$ erstreckt sich von Augusta praetoria, Augst, also Aosta, bis zu Gemmi und Rawyl und von St. Moritz, also St-Maurice, und Martinach (Martigny) bis zur Furka. Auch der Brienzer See ist sichtbar. Das letzte Blatt, Die Rauracer, Maßstab etwa $1: 290$ 000, umfaßt die heutigen Kantone Basel-Stadt und -Land und Teile der Kantone Aargau, Solothurn und Bern; wir finden auch den Sundgau und den Schwarzwald; das Blatt erstreckt sich von Coblenz-Waldshut bis Lützel (kleines Dorf, wie eine Stadt dargestellt) und von Zofingen bis ins Wiesental bei Basel.

Es ist gar nicht zu verwundern, daß der hübsche und nützliche Atlas guten Absatz fand. Die groben Verzeichnungen der damaligen Karten gehen auf das damals übliche rohe Aufnahmeverfahren auf Grund der Reisedistanzen in Schritten oder in Zeit und von Richtungsschätzungen, Messungen mit groben Meßscheiben oder Azimutmessungen mit Bussolen zurück, bei dem sich mit zunehmender Distanz die Fehler häufen mußten. Ernst Winkler hat den Stumpfschen Atlas benützt, um auf Grund desselben eine kurze Schilderung der schweizerischen Kulturlandschaft um 1550 zu entwerfen (Band II der Geographica Helvetica, 1947, Seite 223). 


\section{Geneva Civitas, von Jacques-Barthélemy Micheli du Crest}

Wir haben vor uns eine Karte im Format von $74 \times 54 \mathrm{~cm}$, im Maßstab $53,7 \mathrm{~mm}=300$ Genfer Toisen $=400$ französische Toisen, was einer Verjüngung von $1: 14500$, also rund $1: 15000$ entspricht. Die Karte erstreckt sich von Château de Tournay über Grand-Saconnex nach Cointrin und Vernier, weiter über Loëx und Onex und Veiry bis zum Salève und bis Gaillard und Ambilly. Hecken, Bäume, Wälder und Reben sind äußerst sorgfältig in Grün angegeben, ebenso die Kulturen. Äcker und Wiesen sind im Ton unterschieden. Das Terrain ist in äußerst feinen Schraffen in Vertikalprojektion angegeben. Die Gebäude sind rot eingezeichnet, die Befestigungen der Stadt Genf sorgfältig dargestellt. Höhenzahlen fehlen vollständig. Beschriftung ist sehr wenig, aber sauber und gut lesbar vorhanden. Die Zeichnung wurde offensichtlich mit größter Sorgfalt und viel Liebe ausgeführt. Die Karte zeigt fast genau NNO oben, sie ist also nicht ostorientiert (Grob). Aufgenommen wurde die Karte von Micheli du Crest selber in den Jahren 1721-1727. Über sein Aufnahmeverfahren äußert er sich in einem Brief von 15. Mai 1755 an Albrecht von Haller in Bern. Er hat die Karte als ebenen Plan aufgenommen und zwar, wie wir heute sagen, durch graphische Triangulation, durch Vorwärtseinschneiden mit dem Meßtisch. Ausgehend von den so bestimmten Fixpunkten zeichnete er die Topographie ein mit dem Meßtisch und dem Croquierbrett. Die Karte, die ein kartographisches Meister- und Kunstwerk darstellt, trägt den Namen des Autors nicht, so daß sie gegen das Ende des 19. Jahrhunderts neu identifiziert werden mußte. Die Art und Weise, wie der Plan in den Besitz der Genfer Bibliothek gelangte, wurde von M. Fréd. Gardy dargestellt. M. du Crest hatte sich bekanntlich mit dem Genfer Großen Rat überworfen, dessen Mitglied er seit 1721 selbst gewesen war. Er wurde am 9. Mai 1730 aus dem Rate gestoßen, im Bürgerrecht eingestellt und seine Güter unter Sequester gelegt. Merkwürdigerweise zeigte er daraufhin unter dem 18. Juni 1730 dem Direktor der öffentlichen Bibliothek in Genf, Abauzit, aus Landau, wo er als Offizier diente, an, daß er die Absicht habe, seine schöne Karte von Genf und Umgebung der Bibliothek zu schenken, und ersuchte ihn, ihm mitzuteilen, ob die Bibliothekskommission bereit sei, das Geschenk anzunehmen. M. du Crest wollte sich vielleicht auf diese Weise bei seinen Mitbürgern in vorteilhafte Erinnerung rufen. Die Bibliothekskommission war hocherfreut und nahm das Angebot mit dem gebühren- 
den Dank entgegen, um so mehr, als M. du Crest bereits vorher der Bibliothek eine Anzahl wertvoller Bildnisse geschenkt hatte. M. du Crest adressierte nun das Colis an einen Notar in Genf, der es der Bibliothekskommission hätte übergeben sollen. Der "tabellion» bekam es aber offenbar mit der Angst zu tun, weil M. du Crest ja erst verurteilt worden war. Er benachrichtigte die Behörden von Genf, die unterm 31. Juli 1730 den Notar anwiesen, ihnen das Colis zu übergeben, um darüber zu beraten. Die Behörden suchten die Karte den Blicken des Publikums zu entziehen, um der Erinnerung an M. du Crest nicht neue Nahrung zu geben. Die Bibliothek hatte also vorläufig das Nachsehen. M. du Crest gab aber seine Absicht nicht auf. Er verfolgte die Angelegenheit mit seiner gewohnten Zähigkeit weiter und nahm sogar die Hilfe des Comte de Lautrec, des französischen Vermittlers in den Unruhen von 1738, in Anspruch. Er erreichte auch sein Ziel, indem die Genfer Behörden, die mit der Vermittlung zufrieden gewesen waren, dem Unterhändler sein Begehren nicht abschlagen wollten, so daß sie die Karte der Bibliothek übergaben, nachdem sie dieselbe dem Comte de Lautrec vorgewiesen hatten. Unterm 10. Juni 1738 findet sich der Eintrag des Einganges der Karte in den Registern der Bibliothek. Trotzdem wurde die Karte außerhalb von Genf nicht beachtet, auch nicht von Rud. Wolf, obschon er den weiter oben erwähnten Brief vom 15. Mai 1755 gekannt hat. Es ist das Verdienst von Prof. Dr. J. H. Graf in Bern, auf die Karte aufmerksam gemacht zu haben. Dieselbe wurde 1926 im Auftrage der Genfer Bibliothèque publique und der Genfer geographischen Gesellschaft von der Firma Kümmerly \& Frey in Bern reproduziert. Diese Reproduktion in hundert Exemplaren hat ganz außerordentliche Kosten verursacht, da bei der außerordentlichen Feinheit des Originals und seiner Tönung die Photographie nur die Grundlage liefern konnte und im übrigen die ganze Karte von Hand lithographiert werden mußte. Diese Reproduktion stellt eine späte, aber wohlverdiente Rehabilitation für den unglücklichen Urheber der wunderbaren Karte dar.

Wie aus der Biographie von J. B. Micheli du Crest von Prof. J. H. Graf hervorgeht (Seite 161) sowie aus einer brieflichen Mitteilung von Herrn Kantonsgeometer E. Dériaz vom 11 . September 48, die hier bestens verdankt sei, wurde 1726 in Genf auf Initiative von M. du Crest und nach seinen Instruktionen eine Stadtvermessung unter der Leitung von Ingenieur und Architekt Billon im Maßstab von $1: 240$ ins Werk gesetzt $(1 \mathrm{Zoll}=20 \mathrm{Fuß})$. Die Aufnahmen auf dem Terrain nahmen vier Mo- 
nate in Anspruch, ebensoviel die Reinzeichnung der Pläne (plans Billon), die samt dazugehörigen Dokumenten sich im Genfer Staatsarchiv befinden. Billon erstellte dann noch eine Reduktion dieser Aufnahme, einen Übersichtsplan im Maßstab 1:800 samt einer Kopie desselben. Diese Plans Billon dienten noch 1837 dem Ingenieur Céard, um einen Stadtplan von Genf für die Feuerwehr zu erstellen. Das Beispiel der topographischen und kartographischen Arbeiten von M. du Crest wirkte auch über die Grenzen hinaus, indem dadurch der König Viktor-Amadeus von Savoyen veranlaßt wurde, eine Katastervermessung in Savoyen anzuordnen, die von 1728 bis 1735 durchgeführt wurde. Es sei erwähnt, daß aus den «Confessions» von J. J. Rousseau hervorgeht, daß er auf Veranlassung von Mme. de Warens bei dieser Vermessung eine Zeitlang als Zeichner mitwirkte. Solche Zeichnungen von J. J. Rousseau waren auf der Weltausstellung in Paris 1889 ausgestellt, jedoch scheint Prof. F. Becker, gewiß ein kompetenter Kritiker, nicht gerade davon erbaut gewesen zu sein.

Es muß auch daran erinnert werden, daß Micheli du Crest als erster den Plan einer vollständigen Vermessung der Schweiz gefaßt und ausgearbeitet und denselben bei den bernischen und eidgenössischen Behörden vertreten hat. $\mathrm{Ja}$, er hat noch versucht, soweit ihm dies in seiner unglücklichen Lage als Staatsgefangener in Aarburg möglich war, denselben durch Basismessungen usw. zu realisieren. Der Verständnislosigkeit der damaligen bernischen und eidgenössischen Behörden verdanken wir es, daß der Beginn einer wirklichen Vermessung der Schweiz etwa um Dreivierteljahrhundert hinausgeschoben wurde.

\section{Literatur}

F.Becker, Die schweizerische Kartographie an der Weltausstellung in Paris 1889, Seite 71, Frauenfeld 1890.

J.H.Graf, Jacques Barthélemy Micheli du Crest, Bern 1890.

J.J. Rousseau, Les Confessions.

3. Dufourkarte des Kantons Genf $1: 12500$, Neuausgabe von 1925

Diese Karte wurde auf Veranlassung des damaligen eidgenössischen Oberstquartiermeisters und Kantonsingenieurs G. H. Dufour in den Jahren 1835-1838 unter seiner Leitung auf Kosten des Kantons Genf aufgenommen. Gleichzeitig wurde ein sehr enges Nivellementsnetz ausgemessen, das auf den Bronzefixpunkt auf der Pierre du Niton mit der Kote $376,30 \mathrm{~m}$ bezogen wurde. Von 1835 bis 1837 besorgte Jean Frédéric d'Osterwald 
die Messung und Berechnung der Triangulation. Als Basis für diese Triangulation wurde die Seite Dôle-Bougy der schweizerischen Haupttriangulation verwendet, $d . h$. die Triangulation an die eidgenössische Triangulation angeschlossen, da Dufour diese Kantonsvermessung für die Schweizerkarte 1 : 100000 zu verwenden wünschte. Dr. Hans Zölly, Bern, hat das Netz dieser Triangulation aufgefunden und publiziert. Es hat sich dabei gezeigt, daß Osterwald mit einem Minimum von Signalen eine genügend große Anzahl von Punkten bestimmt hat für die topographische Aufnahme, und zwar rein durch Vorwärtseinschneiden. Das Verfahren hatte aber den Nachteil, daß keine einheitlich ausgeglichene Triangulation erhalten wurde. Sie erwies sich denn auch als genügend genau für die topographische Aufnahme, für die Aufnahme des Katasters jedoch mußte sofort zu einer neuen Triangulation geschritten werden (base de Puplinge). Daß Dufour die Genfer Vermessung als Vorschule zur Landesvermessung betrachtete, erwähnt auch Rudolf Wolf in seiner Geschichte der Vermessungen und geht auch aus einem Votum von Prof. Emile Chaix in der Sitzung der Genfer geographischen Gesellschaft vom 7. Mai 1924 hervor, indem derselbe im Alter von siebzehn Jahren einem diesbezüglichen Gespräch zwischen Prof. Paul Chaix und General Dufour beiwohnte.

Die Karte weist sechzehn Blätter auf im Maßstab 1 : 12500 mit Höhenkurven im Abstand von vier Metern und stellte eine der besten Aufnahmen der damaligen Zeit dar. Sie wurde 1837/38 durch Wolfsberger auf den Maßstab 1 : 25000 reduziert und das Original für die Gravur (Schraffenterrain) erstellt. Die Gravur auf Kupfer wurde durch Rinaldo Bressanini vorgenommen und die reduzierte Karte 1842 publiziert. Diese Schraffenkarte 1:25000 diente als direkte Grundlage für den genferischen Teil der Dufourkarte 1: 100 000. Die Karte 1:12 500 wurde nicht publiziert; die Blätter wurden jedoch derartig intensiv benützt, daß eines davon abhanden gekommen ist, die andern übermäßig abgenützt wurden, ja einige vollständig abgegriffen waren. Die Société de Physique et d'Histoire Naturelle in Genf suchten daher das Werk zu retten durch eine Reproduktion, die ihr vom Staatsrat erlaubt wurde. Es wurde nun aber nicht bloß eine Reproduktion vorgenommen, sondern alle Veränderungen seit der Erstellung der Blätter nachgetragen. Zu dem Zweck mußten die neunhundert Blätter des Katasters photographisch reduziert werden, die Isobathen des Sees nach Pictet (1877) mit fünf Meter Kurvenabstand und diejenigen des Genfer Hafens und der Genfer Reede mit fünfzig Zentimeter Kurvenabstand nach den Angaben des schweizerischen 
Wasserwirtschaftsamtes sowie die Untiefen und die für die Schiffahrt gefährlichen erratischen Blöcke eingezeichnet werden. Die Karte wurde auf diese Weise auf den Stand von Anfang 1924 nachgeführt. Die Reproduktion wurde von der kartographischen Anstalt Kümmerly \& Frey, Bern, in Lithographie vorgenommen. Das Werk präsentiert sich nunmehr als ein Atlas des Kantons Genf im Planmaßstab von 1:12 500, dreifarbig. Eines der Blätter ist vierfarbig, indem Blatt XI, auf welchem die Stadt Genf dargestellt ist, sich in zwei Exemplaren vorfindet. Das eine, dreifarbige Exemplar entspricht dem Stand vom März 1924, das andere, das historische Blatt, auf dem natürlich auch noch die Befestigungen figurieren, stellt die genaue Reproduktion des Originalblattes, vierfarbig, dar. Die Stadt erscheint hier mit den Befestigungen beinahe noch wie auf der Karte von Micheli du Crest. Die geographischen Koordinaten sind auf den Karten nicht angegeben, dagegen ist ein Kilometernetz vorhanden. Auf Blatt XI (historische Ausgabe) ist der Meridian eingezeichnet, der durch die tour à la cloche d'argent de la cathédrale de St-Pierre geht. Er bildet mit der angenähert Nord-Süd verlaufenden Linie des Kilometernetzes durch denselben Punkt den Winkel der Meridiankonvergenz von $55^{\prime} 52^{\prime \prime} \pm 2^{\prime \prime}$ (Angabe der Eidgenössischen Landestopographie), d. h. das Kilometernetz dieser Karte liegt parallel zum Kilometernetz der Siegfriedkarte, entgegen der anders lautenden Angabe auf dem Titelblatt der Neuausgabe der Karte 1:12 500 .

So konnte durch die großen Opfer an Zeit, Arbeit und Geld der beteiligten Fachleute, staatlichen Stellen, wissenschaftlichen Gesellschaften und der kartographischen Anstalt das wertvolle Werk des späteren Generals Dufour nicht nur gesichert, sondern von neuem nutzbar gemacht werden.

Von diesem Atlas 1:12500 erschienen zwei Ausgaben, nämlich eine Ausgabe auf gewöhnlichem lithographischem Papier und eine Luxusausgabe auf Büttenpapier.

\section{Literatur}

H.Zoelly, Les bases géodésiques des mensurations dans le Canton de Genève depuis 1821. Bull. Techn. de la Suisse Romande, No. 22, année 1923. 